\title{
Use of Hybrid Capillary Tube Apparatus on 400 MHz NMR for Quantitation of Crucial Low-Quantity Metabolites Using aSICCO Signal ${ }^{\mathbb{}}$
}

\author{
Ranjeet Tiwari, Deepak Ahire, Hemantha Kumar, Sarmistha Sinha, Siddheshwar Kisan Chauthe, \\ Murali Subramanian, Ramaswamy lyer, Ramakanth Sarabu, and Lakshmikant Bajpai
}

\begin{abstract}
Discovery Analytical Sciences (R.T., H.K., S.K.C., R.S., L.B.) and Pharmaceutical Candidate Optimization (D.A., S.S., M.S.), Bristol-Myers Squibb-Biocon Research Center, Bangalore, India; and Pharmaceutical Candidate Optimization, Bristol-Myers Squibb, Princeton, New Jersey (R.I.)
\end{abstract}

Received June 16, 2017; accepted September 13, 2017

\begin{abstract}
Metabolites of new chemical entities can influence safety and efficacy of a molecule and often times need to be quantified in preclinical studies. However, synthetic standards of metabolites are very rarely available in early discovery. Alternate approaches such as biosynthesis need to be explored to generate these metabolites. Assessing the quantity and purity of these small amounts of metabolites with a nondestructive analytical procedure becomes crucial. Quantitative NMR becomes the method of choice for these samples. Recent advances in high-field NMR ( $>500 \mathrm{MHz}$ ) with the use of cryoprobe technology have helped to improve sensitivity for analysis of small microgram quantity of such samples. However, this type of NMR instrumentation is not routinely available in all laboratories. To analyze microgram quantities of metabolites on a routine
\end{abstract}

basis with lower-resolution $400 \mathrm{MHz}$ NMR instrument fitted with a broad band fluorine observe room temperature probe, a novel hybrid capillary tube setup was developed. To quantitate the metabolite in the sample, an artificial signal insertion for calculation of concentration observed (aSICCO) method that introduces an internally calibrated mathematical signal was used after acquiring the NMR spectrum. The linearity of aSICCO signal was established using ibuprofen as a model analyte. The limit of quantification of this procedure was $0.8 \mathrm{mM}$ with $10 \mathrm{~K}$ scans that could be improved further with the increase in the number of scans. This procedure was used to quantify three metabolites-phenytoin from fosphenytoin, dextrophan from dextromethorphan, and 4-OH-diclofenac from diclofenac-and is suitable for minibiosynthesis of metabolites from in vitro systems.

\section{Introduction}

The importance of identification and characterization of metabolites in early-stage drug development has been highlighted in the guideline on Safety Testing of Drug Metabolites issued by the US Food and Drug Administration Centre for Drug Evaluation and Research (Timmerman et al., 2010, 2016; Briggs et al., 2014). This guidance suggests that metabolites that circulate at $10 \%$ or more of total drug-related material in humans at steady state need to be further characterized in safety studies, which may require reanalysis of IND (Investigational New Drug)enabling toxicity studies with quantitative determination of metabolite levels, or conducting fresh safety studies to match the exposure levels of the metabolites observed in humans. These studies are often conducted in the absence of synthetic metabolite standards. Additionally, proactive assessment of metabolite levels in IND toxicity studies anticipated to be major in humans is a common mitigation strategy.

Advances in mass spectrometry have contributed to the improvements in identification and characterization of metabolites. However, getting the exact structure from a mass spectrum is still challenging. For any liquid chromatography-mass spectrometry (LC-MS)-based quantification assay,

https://doi.org/10.1124/dmd.117.077073

SThis article has supplemental material available at dmd.aspetjournals.org. for example, multiple reaction monitoring, a qualified metabolite reference standard is needed (Pic and Barcel, 2008; Zhou and Yin, 2016). Thus, many unknown metabolites detected in LC-MS profiling experiments at discovery stage cannot be quantified due to lack of suitable reference standards (Scalbert et al., 2009). Synthesis of many metabolite standards is chemically challenging, so alternate strategies such as the biologic synthesis of metabolites followed by their isolation and quantification have become routine in drug discovery (Tao et al., 2009; Huttanus et al., 2016). Certification of these biologically generated metabolites using the classic methods of analysis is challenging because there is very limited quantity available. Therefore, there is a need for a method of quantification of these metabolites that should be sensitive and nondestructive so that recovered sample solutions could be used as reference standard for subsequent analyses.

NMR spectroscopy is becoming an important tool for the quantitation of small and large molecules (Bharti and Roy, 2012; Pauli et al., 2014). NMR has excellent reproducibility and quantitative accuracy (Wishart, 2008; Nagana Gowda and Raftery, 2015). The response of the signal from NMR is proportional to molar concentrations of the analyte and is independent of the type of other atoms present, which makes it a more accurate quantitative technique compared with other traditional analytical methods of quantitation. At the same time, it is a nondestructive technique, so analytes can be recovered after analysis for further use (Simmler et al., 2014).

ABBREVIATIONS: aSICCO, artificial signal insertion for calculation of concentration observed; DMSO, dimethylsulfoxide; DNB, 3,5-dinitrobenzene; ERETIC, Electronic Reference To Access In Vivo Concentrations; HLB, hydrophilic-lipophilic-balanced; HPLC, high-pressure liquid chromatography; LC-MS, liquid chromatography-mass spectrometry; qNMR, quantitative NMR. 
Quantitation with quantitative NMR (qNMR) has been done by utilizing both an internal as well as an external standard (Barding et al., 2012; Bharti and Roy, 2012; Siddiqui et al., 2013; Pauli et al., 2014; Rundlöf et al., 2014; Simmler et al., 2014). There are several criteria for a compound to be used as NMR internal standard, as follows: 1) it should be available in pure form; 2) it should have well-resolved and distinguished signal(s) from the analyte peak of interest; 3 ) it should be inert when mixed with analyte; and 4) it should be nonvolatile (Saito et al., 2009; Weber et al., 2014). Use of external reference standards for qNMR was helpful to overcome a few of these limitations, such as compatibility and signal interference issues because, in this method, standard and analyte sample with the same volume is taken separately in identical precise NMR tubes and a spectrum is acquired separately (Huang et al., 2014).

Another reported method for quantitation with NMR uses Electronic Reference To Access In Vivo Concentrations (ERETIC) signal as a reference. Applications of ERETIC signal on low-resolution instruments are limited due to variation in response factor caused by variation of several experimental factors (Barantin et al., 1997; Farrant et al., 2010; Liang et al., 2010; Esteve et al., 2012; Jung et al., 2016). Another challenge in the ERETIC signal method is the insertion of the reference signal into the acquisition data, which requires specialized hardware and pulse sequences. Walker et al. $(2011,2014)$ reported the use of a mathematically generated artificial signal insertion for calculation of concentration observed (aSICCO) inserted postacquisition as a reference for the quantitation of metabolites. Any region in the analyte spectra can be selected for the insertion of aSICCO reference signal. The magnitude of the inserted signal can be modified to appropriately match the intensity of the sample resonances. The major limitation of Walker's aSICCO signal technique is that it uses high field magnets $(500 \mathrm{MHz}$ and above with respect to ${ }^{1} \mathrm{H}$ frequency) and requires high-sensitivity probe such as microprobe or cryoprobe.

Another major challenge in the metabolite identification and quantification is the choice of appropriate NMR solvents to receive better resolution of signals. Use of incorrect solvent may lead to improper signal with poor resolution or merging peaks. Generally, metabolite samples are very limited in quantity and difficult to recover and reuse, so there is no opportunity to try different solvents to get the best possible resolution.

For improving the sensitivity in NMR experiments, use of Shigemi NMR tubes has been reported (Krunić and Orjala, 2015) on $900 \mathrm{MHz}$ instrument with cryoprobe. These tubes are matched in terms of magnetic susceptibility to each of the solvents with which they are identified. However, there are certain limitations with these tubes. These can only be used for the specified solvent, reported for the high-sensitivity NMR instruments, available only until 3-mm diameter, have no option to add another solvent for screening, are very costly, and do suffer with appearance of air bubbles between the plunger and the solvent at high temperature, which tends to destroy the field homogeneity (Wieruszeski et al., 2008). To extend the application of the aSSICO signal method for metabolite quantitation, we have developed a novel hybrid capillary tube setup that could be used on $400 \mathrm{MHz}$ NMR instrument with a room temperature broad band fluorine observe probe. This configuration could also be used to screen multiple NMR solvents for structure elucidation of crucial small-quantity metabolites using the same sample without recovery or contamination with uncommon solvents.

\section{Materials and Methods}

Chemicals and Reagents. Analytical grade fosphenytoin, dextromethorphan, and diclofenac drug standards were purchased from Sigma-Aldrich (St. Louis, MO). High-pressure liquid chromatography (HPLC) grade solvents and chemicals such as acetonitrile, methanol, formic acid, malic acid, potassium dihydrogen phosphate $\left(\mathrm{KH}_{2} \mathrm{PO}_{4}\right)$, dipotassium hydrogen phosphate $\left(\mathrm{K}_{2} \mathrm{HPO}_{4}\right)$, and Tris (hydroxymethyl) amino methane (Tris buffer) were purchased from Merck Specialties Private (Mumbai, India). NADPH was purchased from SISCO Research Laboratory (Mumbai, India). Recombinant cytochrome P450s (2D6 and 2C9) were purchased from Corning Life Sciences (Gurgaon, India). The recombinant alkaline phosphatase microsome was prepared by in-house methods (Subramanian et al., 2013). Oasis hydrophilic-lipophilic-balanced (HLB) cartridges were purchased from Waters (Bangalore, India). All deuterated solvents such as dimethylsulfoxide (DMSO)-d6, benzene-d6, and $\mathrm{CDCl}_{3}$ were purchased from Eurisotop (Saclay, France). All other solvents and reagents were highest grade commercially available materials and were used without further purification.

Equipment. The following equipment was used in these preparations, unless otherwise noted: Eppendorf 5810 R centrifuge (North Ryde, Australia); Turbovap LV nitrogen evaporator (Caliper Life Sciences, Hopkinton, MA); HLB cartridgesOasis HLB 1-cc extraction cartridges (Milford, MA); $100 \mu 1$ X-type syringe (CTC Analytics AG, Zwingen, Switzerland); Cyclo-Mixer (Remi Laboratory Instruments, Mumbai, India); HPLC column SunFire C18 (Waters, Milford, MA); and HPLC column Inetrsil-ODS (GL Sciences, Tokyo, Japan).

Preparation of Calibration Standards and Internal Standard Solution. In this study, ibuprofen was used as model analyte for checking the calibration and linearity of aSSICO and traditional qNMR methods. 3,5-Dinitrobenzene (DNB) was used as an internal standard for quantification using the traditional method, whereas maleic acid was used for external calibration using the aSICCO method. DNB is a nonvolatile compound, soluble in a variety of solvents common to drug metabolism studies, and has a sharp resonance at $\delta 8.9 \mathrm{ppm}$. A stock solution of DNB and ibuprofen was prepared by dissolving 6.12 and $10.6 \mathrm{mg}$ solid in 5.00 $(5.77 \mathrm{mM})$ and $2.00 \mathrm{ml}(25.7 \mathrm{mM})$ DMSO-d6, respectively. A series of five standard solutions ranging from $0.80(8.24 \mu \mathrm{g})$ to $12.85 \mathrm{mM}(132.68 \mu \mathrm{g})$ concentration of the ibuprofen was prepared by serial dilution of the stock solution with DMSO-d6. Each sample contained a $2.35 \mathrm{mM}$ DNB acid as an internal standard. All solutions were vortexed and stored in the refrigerator when not in use.

Generation and Calibration of aSICCO Signal. NMR-SIM (Version 3.2, TopSpin, Bruker, Fällanden, Switzerland) was used to create an aSSICO signal. A single-pulse sequence ( $\mathrm{zg}$ ) with uncoupled resonance was used to generate an artificial signal with 1 second as relaxation time. The chemical shift of the artificial signal was decided based on the spectral data of the metabolite of interest. A line broadening of $1 \mathrm{~Hz}$ was used to reproduce the processing of the isolated sample. Before each experiment, the aSSICO signal was calibrated with a known concentration of a standard solution with constant parameters set for both known standard solution and unknown sample data collection. Phase and baseline were manually corrected, and the aSSICO signal and signal from standard were integrated equally. An aSSICO signal relative concentration was assigned using the equation below (Walker et al., 2011):

$$
\text { Conc of aSSICO signal }=\text { Conc. of Std } \times \frac{\mathrm{IC}}{\mathrm{IS}} \times \frac{\mathrm{NS}}{\mathrm{NC}}
$$

where IC = integral of aSSICO signal, IS = integral of standard, NS = number of protons in standard, and $\mathrm{NC}=$ number of protons in sSSICO signal.

The acquisition and processing parameters such as pulse length, relaxation delay, and sweep width were kept identical for both analyte and the calibration data. After generating an artificial signal and calibration data set, both the data points were merged with the help of macro in TopSpin. A scaling factor that is variable within the macro is used to set aSSICO signal similar to that of resonance signal. The chemical shift variable was also available in macro, which allowed setting of aSSICO signal chemical shift value as per the profile of the analyte.

General Method for In Vitro Incubations to Generate Metabolites. In the general method, the parent compound (typically 30-100 $\mu \mathrm{M}$ ) was incubated in an in vitro system containing a source of drug-metabolizing enzymes and cofactors necessary to effect the biotransformation of interest (Table 1). The reaction volume was typically a multiple of $1 \mathrm{ml}$, depending on the requirement and turnover of the compound in the given matrix. All of the incubations were performed at $37^{\circ} \mathrm{C}$ in a shaking incubator at $60 \mathrm{rpm}$. At the end of the incubation, the multiple reaction samples were pooled and passed through Oasis HLB cartridges to remove the matrix interference and concentrate the metabolite(s) samples. A vendor (Waters) recommended protocol was used to process the samples through HLB (http://www.waters.com/webassets/cms/library/docs/ 720005140en.pdf). Approximately $10 \mu \mathrm{l}$ pooled sample was loaded on the 
TABLE 1

In vitro incubation conditions for generation of metabolites of interest

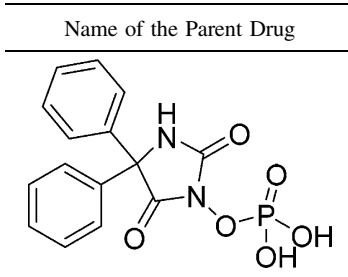

Fosphenytoin<smiles>COc1ccc2c(c1)[C@@]13CCC[C@@]4(CCC[C@]1(C2)N4C)[C@@H]3C</smiles>

Dextromethorphen<smiles>O=C(O)Cc1ccccc1Nc1c(Cl)cccc1Cl</smiles>

Diclofenac<smiles>O=C1NC(=O)C(c2ccccc2)(c2ccccc2)N1</smiles>

Phenytoin

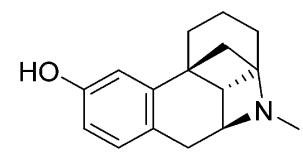

Dextrorphan<smiles>O=C(O)Cc1ccc(O)cc1Nc1c(Cl)cccc1Cl</smiles>

4-OH diclofenac
rCYP2D6 $(0.5 \mathrm{mg} / \mathrm{ml})$

$50 \mu \mathrm{M}$

$\mathrm{KH}_{2} \mathrm{PO}_{4}(100 \mathrm{mM}, \mathrm{pH} 7.4)$

rCYP2C9 $(0.5 \mathrm{mg} / \mathrm{ml})$

$50 \mu \mathrm{M}$

$\mathrm{KH}_{2} \mathrm{PO}_{4}(100 \mathrm{mM}, \mathrm{pH} 7.4)$
Orbitrap for the confirmation of the metabolite formation. The eluted samples from HLB cartridge were subjected to nitrogen evaporation for reducing the sample volume. Resulting concentrated samples were purified on analytical HPLC column. This general method for in vitro incubations to generate metabolites was applied for fosphenytoin, dextromethorphan, and diclofenac drug standards. The details of type of microsome and buffer conditions are given in Table 1.

HPLC Conditions. Concentrated fractions from SPE (Solid Phase Extraction) after incubation were purified on Agilent 1200 series chromatographic system (Santa Clara, CA) equipped with a high-pressure quaternary pump with PDA (Photo Diode Array) detector, heated column thermostat, and a Rheodyne universal loop injector with $100 \mu 1$ injection capacity. Sample injection and data processing were controlled by Chemstation software. The purification of fosphenytoin, dextromethorphan metabolites was carried out on Inertsil-ODS $(250 \times 4.6 \mathrm{~mm}, 5 \mu \mathrm{m})$ column. The mobile phase consisted of $10 \mathrm{mM}$ ammonium acetate buffer in water (solvent A) and acetonitrile (solvent B) at a flow rate of $1 \mathrm{ml} / \mathrm{min}$. The gradient started with $20 \% \mathrm{~B}$ and ramped linearly to $100 \% \mathrm{~B}$ over 25 minutes, held at $100 \%$ B for 5 minutes, returned to the initial condition over
1.0 minute, and allowed to equilibrate for 4.0 minutes. Diclofenac metabolites were purified on SunFire C18 $(250 \times 4.6 \mathrm{~mm}, 5 \mu \mathrm{m})$ column. The mobile phase consisted of $0.1 \%$ trifluoroacetic acid buffer in water (solvent A) and acetonitrile (solvent B) at a flow rate of $1 \mathrm{ml} / \mathrm{min}$. The gradient started with $10 \% \mathrm{~B}$ and ramped linearly to $100 \%$ B over 25 minutes, held at $100 \%$ B for 5 minutes, returned to the initial condition over 2.0 minutes, and allowed to equilibrate for 4.0 minutes. All the metabolites were monitored at 220 and $254 \mathrm{~nm}$ throughout the run time. During chromatography, the column temperature was maintained at room temperature. Fractions containing the metabolite(s) of interest were combined, and the organic solvent removed by nitrogen evaporation and the remaining aqueous sample was passed through a HLB cartridge for a second time, following the same protocol. The eluted organic sample that passed through the HLB cartridge was analyzed on the Orbitrap before nitrogen evaporation to verify the sample integrity. Remaining organic samples were dried under nitrogen.

NMR Sample Preparation and Analysis. After SPE purification, the Eppendorf tubes containing the dried samples were left under vacuum for a minimum of 1 hour. All of the samples were reconstituted in $50 \mu$ l high purity

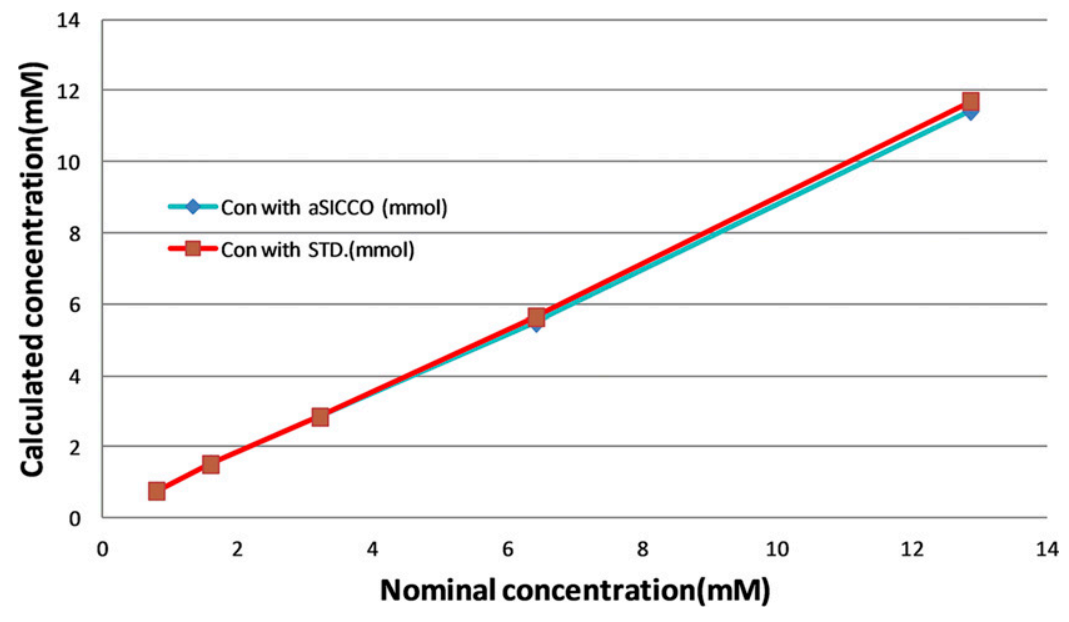

Fig. 1. Comparative analysis of linearity and dynamic range of the classic internal standard qNMR method and the aSICCO method. 


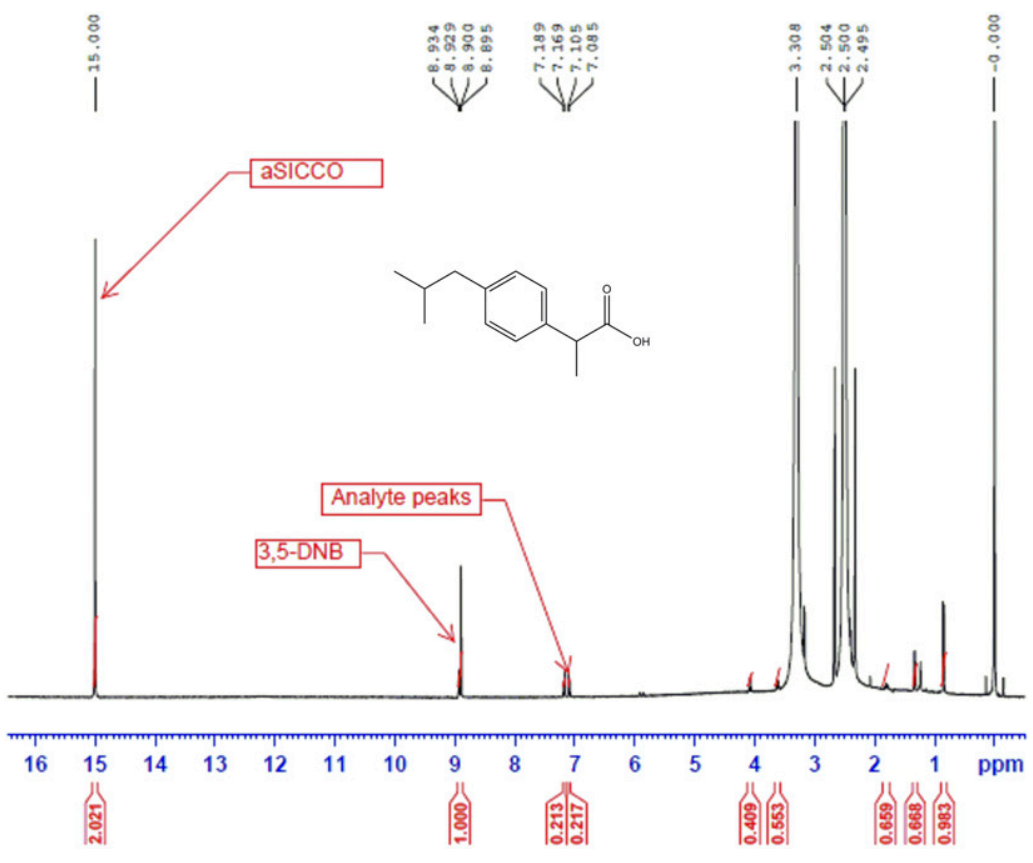

Fig. 2. One-dimensional ${ }^{1} \mathrm{H}$ NMR spectra at $\mathrm{LOQ}$ of $0.8 \mathrm{mM}(8.24 \mu \mathrm{g})$ ibuprofen and $2.35 \mathrm{mM}$ DNB with aSSICO signal.

deuterated DMSO (typically 100\% grade DMSO-d6, which is $99.96 \%$ deuterium enriched) solvent. After addition of solvent, sample tubes were sonicated for 2 minutes and vortexed on cyclomixer for 10 minutes. The resulting clear solution was transferred to the melting-point capillary tube with the help of $100 \mu \mathrm{l}$ microsyringe. After filling capillary tube to two thirds of its size $(50 \mu \mathrm{l})$, it was carefully placed inside the regular 5-mm NMR tube filled with $600 \mu \mathrm{l}$ desired locking NMR solvent.

NMR spectra were recorded on Bruker 400-MHz spectrometer (Bruker BioSpin, Billerica, MA) controlled by TopSpin software (version 3.2) and equipped with a 5-mm broad band fluorine observe probe. One-dimensional proton spectra were recorded with a sweep width of $12,000 \mathrm{~Hz}, 32 \mathrm{~K}$ data point, and a relaxation delay of 2 seconds. For calibration sample and the metabolite samples, $10 \mathrm{~K}$ and $15 \mathrm{~K}$ scans were accumulated, respectively. All spectra were referenced using residual DMSO-d6 $\left({ }^{1} \mathrm{H}, \delta 2.5 \mathrm{ppm}\right)$. Phasing, baseline correction, and integration were all performed manually. qNMR was performed using the aSICCO method (Walker, et al., 2011). For aSICCO method, a standard of known concentration of maleic acid was used as a calibrant. The concentration of the analyte was calculated as per the formula given below:

$\mathrm{mM}$ of Analyte $/$ Conc of aSSICO signal $=\mathrm{mM}$ of IS $/$ Conc of Std $\times \frac{\mathrm{IA}}{\mathrm{IS}} \times \frac{\mathrm{NS}}{\mathrm{NA}}$

IA = Integration of analyte protons (aSICCO signal)/integral of aSSICO signal.

IS = Integration of internal standard protons/integral of standard.

$\mathrm{NA}=$ Number of protons in analyte/number of protons in standard.

$\mathrm{NS}=$ Number of protons in internal standard/number of protons in sSSICO signal.

Quantitation of 4-Hydroxy Diclofenac Metabolite by LC/MS/MS (Liquid Chromatography/Tandem Mass Spectrometry). All prepared samples were analyzed using an Acquity UPLC system coupled to an API 5400 quadrupole ion trap (Applied Biosystems, Toronto, Canada). The chromatographic separation of analytes was achieved on an Acquity BEH C18 column $(2.1 \times 50 \mathrm{~mm}, 1.7 \mu \mathrm{m})$ using a binary gradient mobile phase consisting of $0.1 \%$ formic acid water (mobile phase A) and acetonitrile (mobile phase B) with a flow rate of $800 \mu \mathrm{l} / \mathrm{min}$. Initially, the column was held at $95 \%$ of A for 0.8 minute and then linearly ramped to $50 \%$ of B over 0.4 minute, followed by linear ramping to $90 \%$ of B over 0.4 minute, held at these conditions for next 0.5 minute. The system was then returned to initial conditions and equilibrated for the next 0.5 minute. The total chromatographic run time was 3 minutes. Mass-spectroscopic analysis was performed with an electrospray correct. ionization source operated in negative ion mode with multiple reaction monitoring. The electrospray ionization source temperature was set at $6000^{\circ} \mathrm{C}$, and ion spray voltage, cell entrance (EP-Entrance and Exit Potential), and exit potentials were set at $-4500,-10$, and $-10 \mathrm{~V}$, respectively. The curtain gas, nebulizer gas (Gas 1), and heater gas (Gas 2) were set at 40,30 , and 40 , respectively. The quantification of analyte was achieved by monitoring $\mathrm{m} / \mathrm{z} 312.2 \rightarrow 230.2$ (DP -70 and $\mathrm{CE}-35$ ) for $4-\mathrm{OH}$ diclofenac. The Analyst software (version 4.2) by Applied Biosystems was used for data acquisition and data analysis.

\section{Results}

Ibuprofen was selected as a model analyte for comparing the linearity and dynamic range of classic internal standard qNMR method using DNB as an internal standard versus the aSICCO signal as internal standard. DNB was selected as an internal standard due to its easy commercial availability. ${ }^{1} \mathrm{H}$ NMR spectra of ibuprofen dissolved in DMSO-d6 were recorded at various concentrations from $0.8(8.24 \mu \mathrm{g})$ to $12.85 \mathrm{mM}(132.68 \mu \mathrm{g})$. For each spectrum, $10 \mathrm{~K}$ scans were acquired. Each sample contained $2.35 \mathrm{mM} \mathrm{DNB}$ as an internal standard. For classic qNMR method using DNB as an internal standard, the linearity calculations were performed using signals at $\delta 7.10$ and $\delta 8.90$ for the aromatic protons of ibuprofen and the internal standard DNB,

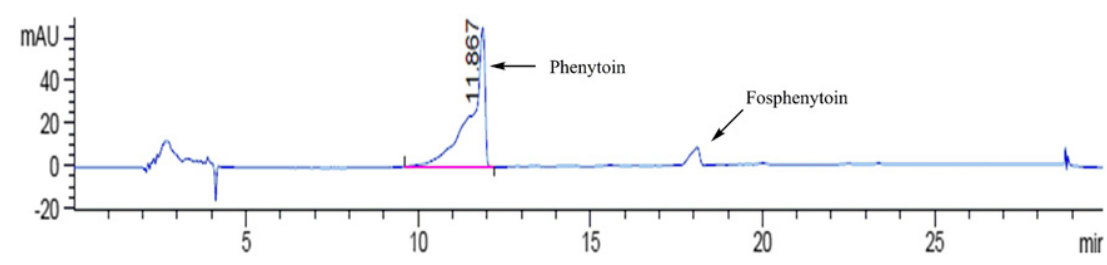

Fig. 3. HPLC UV chromatogram of fosphenytoin metabolites depicting phenytoin as single major metabolite. 


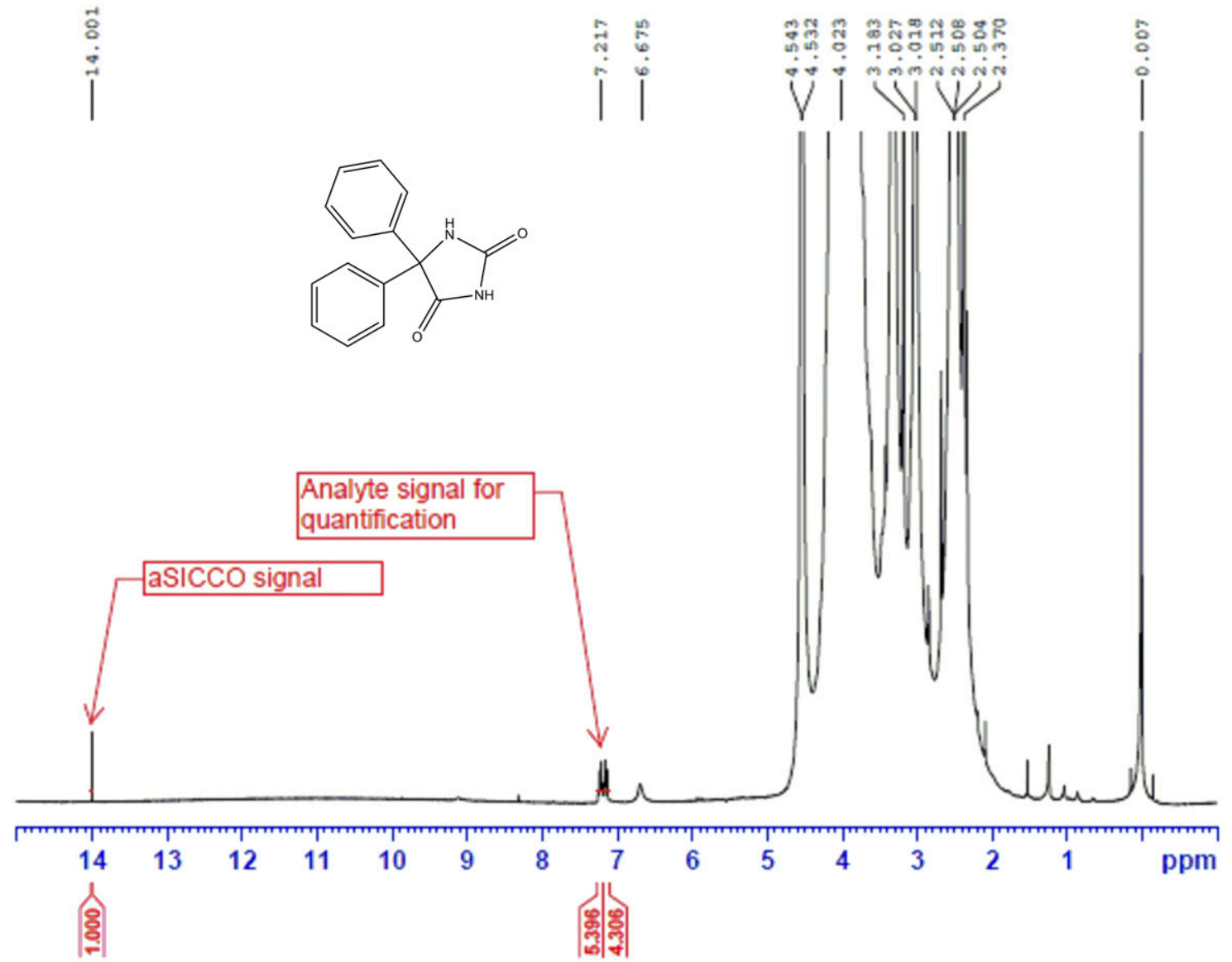

Fig. 4. ${ }^{1} \mathrm{H}$ NMR of phenytoin depicting analyte signals in the aromatic region and aSICCO signal at $\delta 14 \mathrm{ppm}$.

respectively. For the aSICCO method, the signals at $\delta 7.10$ and $\delta 15.0$ for aromatic proton of ibuprofen and artificially inserted aSICCO signal were used for linearity calculations, respectively. In both cases, the response was linear with correlation coefficients greater than 0.999 and intercepts not significantly different from 0 (Figs. 1 and 2).

To demonstrate the utility of the aSICCO approach, quantitative estimation of major metabolites of fosphenytoin, dextromethorphan, and diclofenac drug was performed using aSICCO as reference standard with $15 \mathrm{~K}$ scans so as to improve the sensitivity for the metabolite signal. Maleic acid standards used to calibrate the aSICCO signal were also acquired with $15 \mathrm{~K}$ scans. Reported methods of incubations were used to generate major metabolite for each of these drugs, and metabolites were then isolated by SPE and HPLC chromatography and were concentrated for NMR analysis.

Phenytoin Metabolite. Fosphenytoin is a phosphate ester derivative of phenytoin that is susceptible to hydrolysis and forms phenytoin as a major metabolite (Fischer et al., 2003). Fosphenytoin was incubated with recombinant alkaline phosphatase to generate phenytoin as a single metabolite (Fig. 3; Supplemental Fig. 1). As per the UV chromatogram,
$90 \%$ conversion of parent to phenytoin was observed. The isolated metabolite was confirmed by matching the retention time and mass with the known standard. The overall recovery of phenytoin metabolite was found to be $84 \%$ on w/w basis. The phenytoin metabolite has been previously characterized using ${ }^{1} \mathrm{H}$ NMR, mass spectrometry, and tandem mass spectrometry techniques (Annesley et al., 2001). Crude material obtained after drying was reconstituted in DMSO-d6, and ${ }^{1} \mathrm{H}-\mathrm{NMR}$ was recorded; simultaneously, $0.45 \mathrm{mM}$ maleic acid standard in DMSO-d6 was also acquired to calibrate the aSICCO signal (Fig. 4). The aromatic proton of phenytoin at $\delta 7.1$ with integral of 4.3 and aSICCO signal at $\delta 14$ with integral of 1 was used for qNMR calculations. The calculated concentration of the isolated metabolite was found to be $1.5 \mathrm{mM}$ by the aSICCO qNMR method.

Dextrorphan Metabolite. Dextromethorphan is rapidly converted into the active metabolite dextrorphan by the cytochrome P450 enzyme CYP2D6 (Schadel et al., 1995). After in vitro incubation using the general procedure described in experimental section, dextrorphan was isolated using analytical HPLC (Fig. 5). As per the UV chromatogram, $65 \%$ conversion was observed for dextrorphan metabolite from the

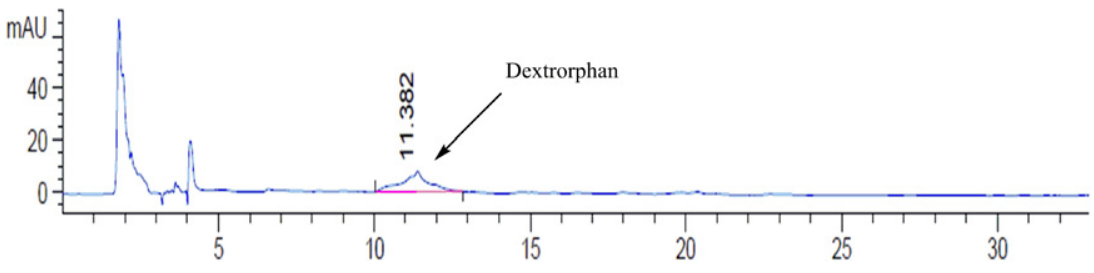

Fig. 5. HPLC UV chromatogram of dextromethorphan depicting dextrorphan as a single major metabolite. 


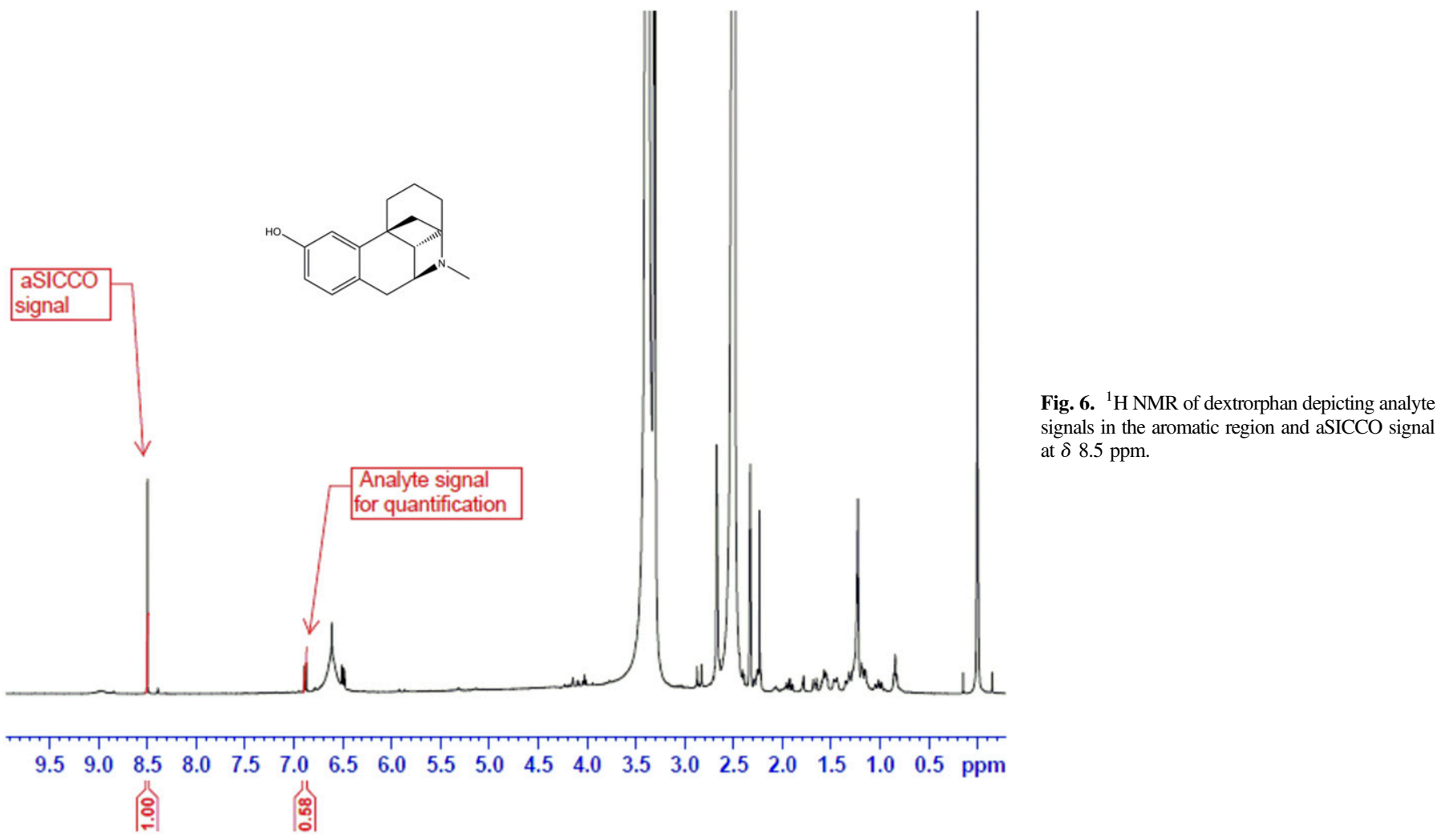

parent drug during in vitro incubation (Supplemental Fig. 2). The isolated metabolite was confirmed by matching the retention time and mass with the known standard. The overall recovery of pure dextrorphan metabolite was found to be $69.7 \%$ on w/w basis. ${ }^{1} \mathrm{H}$ NMR was recorded for the isolated metabolite in DMSO-d6. Simultaneously, ${ }^{1} \mathrm{H}$ NMR of $8.14 \mathrm{mM}$ maleic acid standard was also acquired to calibrate the aSICCO signal (Fig. 6). The aromatic proton of dextrorphan at $\delta 6.8$ with integral of 0.58 and aSICCO signal at $\delta 8.5$ with integral of 1 were used for qNMR calculations. The calculated concentration of the isolated metabolite was found to be $3.33 \mathrm{mM}$ by qNMR using the aSICCO method.

4-Hydroxy Diclofenac. Diclofenac was metabolized to 4-hydroxy diclofenac by polymorphic CYP2C9 (Dorado et al., 2003). After in vitro incubation using general procedure described in experimental section, 4-hydroxy diclofenac was isolated using analytical HPLC (Fig. 7). As per the UV chromatogram, $80 \%$ conversion was observed for 4-hydroxy diclofenac from the parent drug during in vitro incubation (Supplemental Fig. 3). The overall recovery of pure 4-hydroxy diclofenac was found to be $76 \%$ on w/w basis.
${ }^{1} \mathrm{H}$ NMR was recorded for the isolated metabolite in DMSO-d6. Simultaneously, ${ }^{1} \mathrm{H}$ NMR was acquired for $7.28 \mathrm{mM}$ maleic acid standard to calibrate the aSICCO signal (Fig. 8). The aromatic proton of 4-hydroxy diclofenac at $\delta 6.1$ with integral of 1 and aSICCO signal at $\delta 14$ with integral of 1.17 was used for qNMR calculations. The calculated concentration of isolated metabolite was found to be $4.13 \mathrm{mM}$ by qNMR using the aSICCO method.

\section{Discussion}

The utility of NMR for metabolite structural characterization has been well established (Caceres-Cortes and Reily, 2010; Forseth and Schroeder, 2011). In recent years, with the advent of high-field instrument and availability of cryo probes, the ability of NMR to quantify metabolite in low microgram to nanogram quantities is possible (Spraul et al., 2003; Molinski, 2010). However, these types of high-end instrumentation are not available in all of the laboratories. The work presented in this study provides some practical solution to utilizing a $400 \mathrm{mHz}$ NMR with a 5-mm probe to conduct metabolite characterization

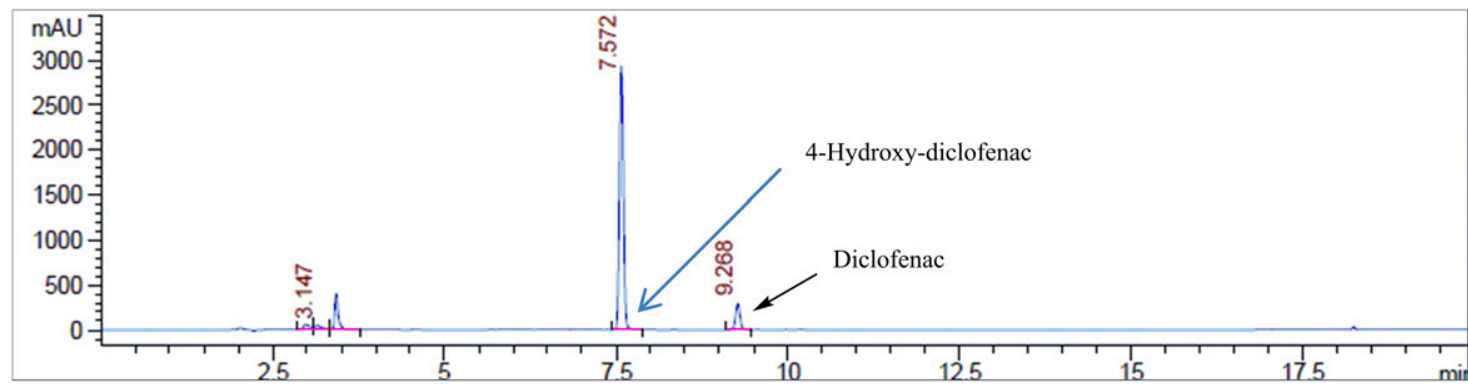

Fig. 7. HPLC UV chromatogram of diclofenac depicting 4-hydroxy diclofenac as a single major metabolite. 


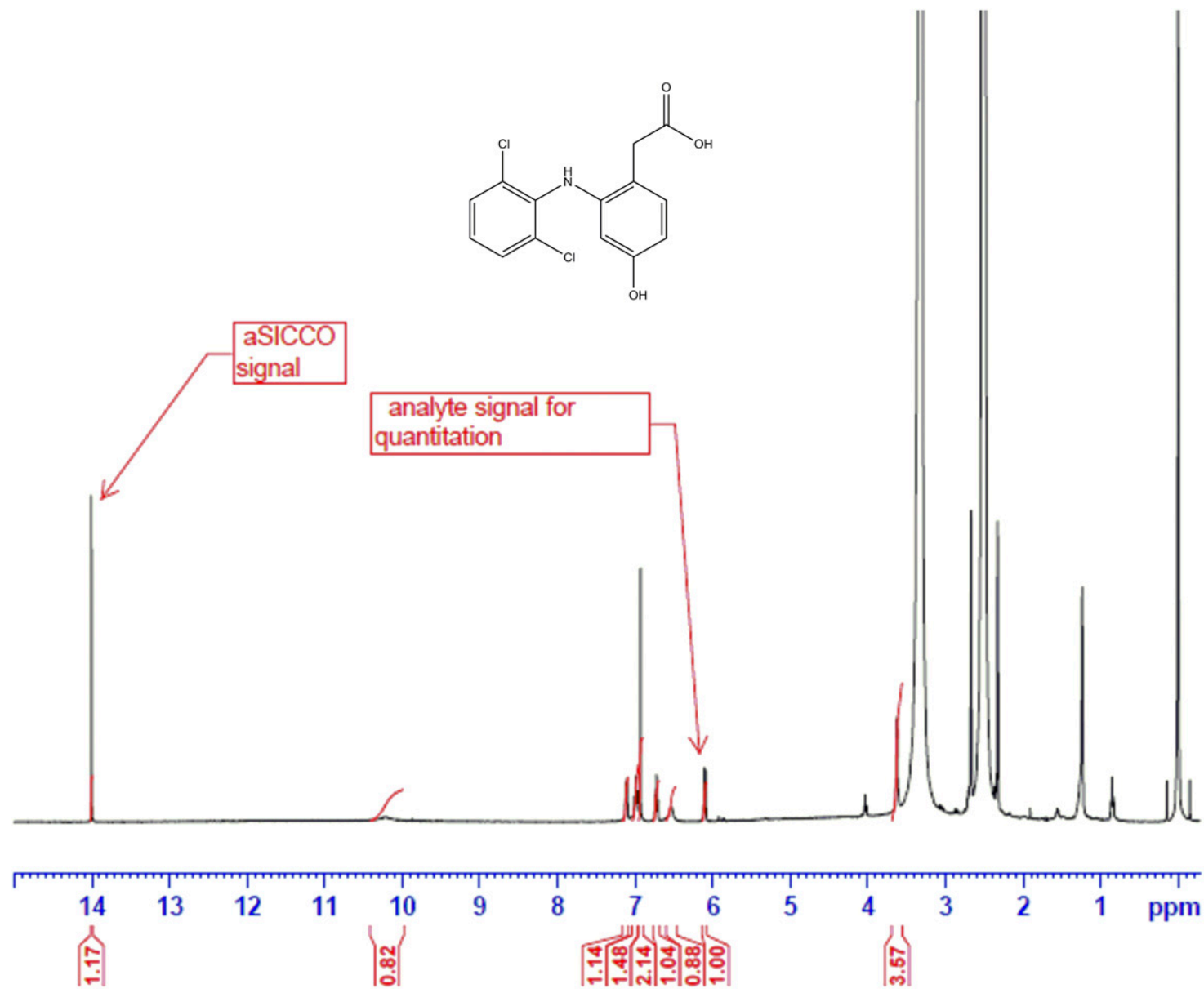

Fig. 8. ${ }^{1} \mathrm{H}$ NMR spectrum of 4-hydroxydiclofenac depicting analyte signals in the aromatic region and aSICCO signal at $\delta 14 \mathrm{ppm}$.

and quantitation. A major limitation for the quantitation of drug metabolites with a $400 \mathrm{MHz}$ NMR instrument is sensitivity, as the amount of metabolite generated from in vitro incubations is typically in the range of microgram to nanogram quantities.

Sample concentration could be enriched by carrying out multiple incubation reactions, but isolating the required metabolite from large incubation volumes is a challenging task. Another way of increasing the sensitivity would be the use of microprobe designed for small volume samples, such as a 1-mm probe, in which sensitivity is increased by improved filling factors. Another advantage of such a probe is that the smaller sample volume $(\sim 12 \mu \mathrm{l})$ reduces the interference noise from the NMR solvent and residual water, which in turn enhances the analyte signal-to-noise (Schlotterbeck et al., 2002). However, this option requires dedicated high-cost probes, which may be a limiting factor in many laboratories. Earlier reports proved that the use of $1.7-\mathrm{mm}$ id (internal Diameter) NMR tube for metabolite analysis was helpful to reduce the interference/noise from NMR solvent and residual water, yet the sensitivity gain from using a smaller diameter NMR tube in a 5-mm probe was insignificant because the rf (radio-frequency) coil was further away from the sample and interference from the air was compensating all the gain (Schlotterbeck et al., 2002; Johnson et al., 2010). Based on this available information, a unique hybrid capillary tube setup was developed that uses a 1-mm capillary tube within a 5-mm outer NMR tube, in a 5-mm room temperature probe. With this strategy, capillary

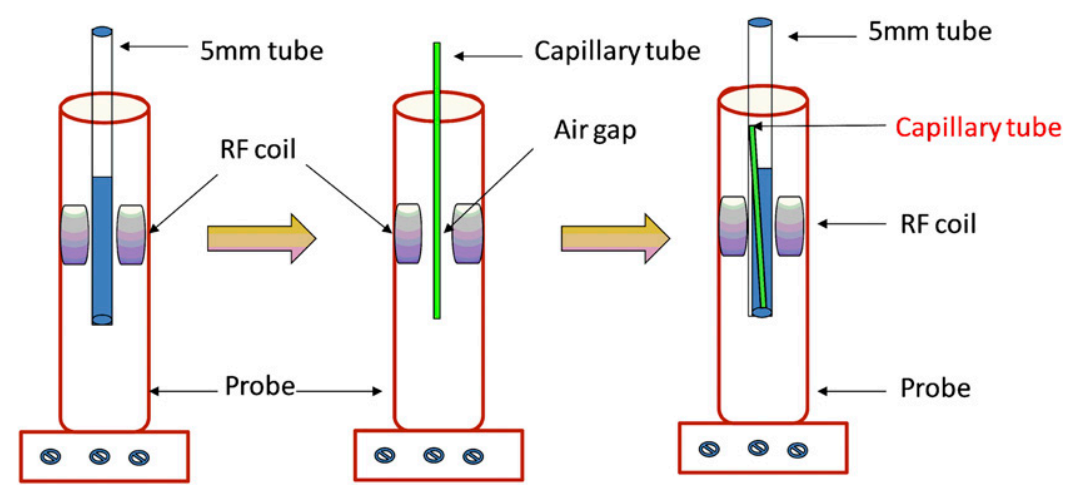

Fig. 9. Pictorial representation for evolution of hybrid capillary tube apparatus in 5-mm NMR broad band fluorine observe probe. 


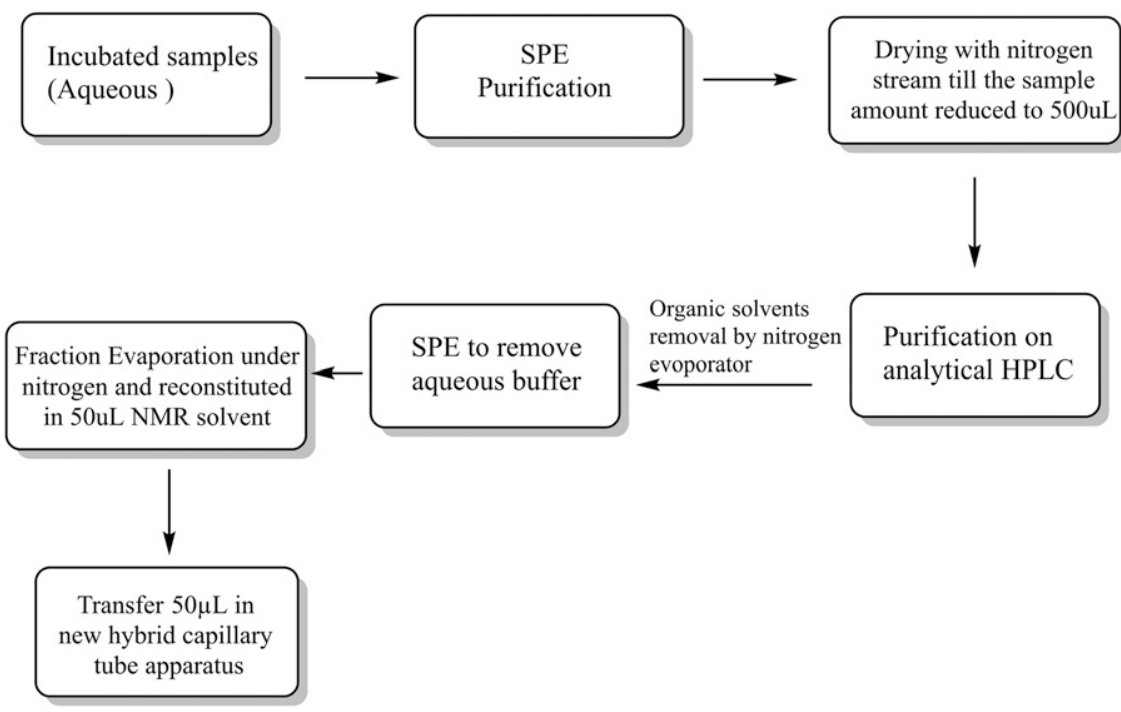

Fig. 10. Schematic diagram showing steps followed in final optimized conditions in isolation and purification of metabolites. tubes were used as sample reservoir needing only 20-30 $\mu$ l sample solution compared with 600-700 $\mu \mathrm{l}$ sample in 5-mm tubes with data acquired on a 5-mm probe. Practically this should increase the actual sample concentration and sensitivity by multiple folds, but increased noise was observed in the spectra collected probably created by the air gap between the rf coil and the sample solution (Fig. 9). To reduce the noise due to the air gap, the capillary tube containing 20-30 $\mu$ l sample solution in deuterated solvent was placed inside a 5-mm tube containing $600 \mu \mathrm{l}$ same NMR solvent (Fig. 9). This theoretically should reduce the interference due to air gap between the rf coil and the sample while maintaining the sensitivity gain due to increased sample concentration. To confirm this, NMR spectra of ibuprofen was acquired in DMSO-d6 in capillary tube inserted in two separate 5-mm NMR tubes: one containing DMSO in the outer tube, and other without solvent in the outer tube. Signal-to-noise ratio was found to be 301 when the outer tube was filled with DMSO and 126 (Supplemental Figs. 4, A and B, and 5) when outer tube was empty, which supported the proposed hypothesis.

Linearity samples were acquired using 2-second relaxation delay with 10,000 scans, and it was observed that the integration values for all of the characteristic signals were linear. For a fully relaxed proton, acquisition time + delay time should be $>5 \times \mathrm{T} 1$ for the resonances being measured (Edwin, 2000). T1 for the test analyte ibuprofen was measured and was found to be 1.3 seconds. Acquisition time for this experiment was 1.99 seconds, and delay time was 2 seconds under the experimental conditions used, which suggested a total of relaxation delay as three times of T1. Usually signal amplitude drops as the recycle time (relaxation + acquisition time) gets shorter, but there are two choices to compensate the loss, as follows: 1) either to cut down recycle time and accumulate more scans with a lower signal recovery for each scan, or 2) use a longer recycle time and fewer scans, with each scan recovering nearly $100 \%$ of the signal. Increasing the relaxation delay would have increased the total run time significantly, so loss of signal amplitude with lower relaxation delay was compensated by increasing the number of scans to 15,000 for metabolite samples and the aSICCO standards with 2-second relaxation delay, and the total acquisition time was 21 hours. To confirm whether 2 seconds of delay time would have major impact on the quality of the data, we have acquired two data at 2- and 10-second relaxation delay, and the signal-to-noise ratios in both cases were found to be 1912 and 1989, respectively (Supplemental Figs. 6 and 7). Even relative integration of all protons was also similar under both conditions, as shown in Supplement Figs. 6 and 7. Similar parameters were used for both analytes and the standard used for calibrating the aSICCO signal, so effective loss in signal amplitude due to shorter relaxation delay should be compensated.

To avoid breaking of the capillary tube, the NMR acquisitions were performed without rotation of the sample tube. With this new hybrid capillary tube appratus, we were able to achieve the quantitation limit up to low microgram level on $400 \mathrm{MHz}$ instrument with room temperature probe. To test the utility and accuracy of this hybrid capillary tube apparatus, quantitation of ibuprofen was performed with a standard $400 \mathrm{MHz}$ NMR using the aSICCO approach. ${ }^{1} \mathrm{H}$ NMR spectra of ibuprofen at different concentrations were acquired using identical instrumental parameters. In all cases, the response of the analyte was linear.

Traditionally, the incubated samples are being purified using chromatographic techniques prior to NMR acquisition (Walker et al., 2014). However, prior to introducing the samples onto the column, it needed to be concentrated. Initial attempts to concentrate the sample by rotary evaporation, Genevac, or lyophilization led to degradation of metabolites (Supplemental Fig. 8). Apparently, the presence of water in the sample took much longer time to concentrate, which caused the degradation. It was confirmed by LC-MS analysis of the samples during multiple stages of purification. To avoid this degradation, after incubation the sample was passed through SPE cartridges to remove the biologic matrix and the water from the sample. A similar SPE concentration step was introduced after the collection of HPLC fraction to remove the water and buffer. Eluent from SPE was dried down under nitrogen, and final residue was reconstituted in $50 \mu \mathrm{l}$ NMR solvent for acquisition (Fig. 10). Overall sample recovery in this process was found to be between $76 \%$ and $86 \%$. SPE steps were not completely optimized for this study as we used the commercial samples, and it is well known that recovery from SPE is compound dependent and can vary from sample to sample. Therefore, there is ample opportunity to further improve the sensitivity for actual study samples by optimizing the SPE step and thereby improve the overall recovery.

Three drugs, fosphenytoin, dextromethorphan, and diclofenac, were incubated to generate the specified metabolites for quantification, as per the protocol specified in experimental section. The corresponding metabolites phenytoin, dextrorphan, and 4-hydroxy diclofenac were isolated with the optimized protocol described above. qNMR was performed with the aSSICO method for metabolite quantitation. The calculated concentration of 4-hydroxydiclofenac obtained by aSICCO 


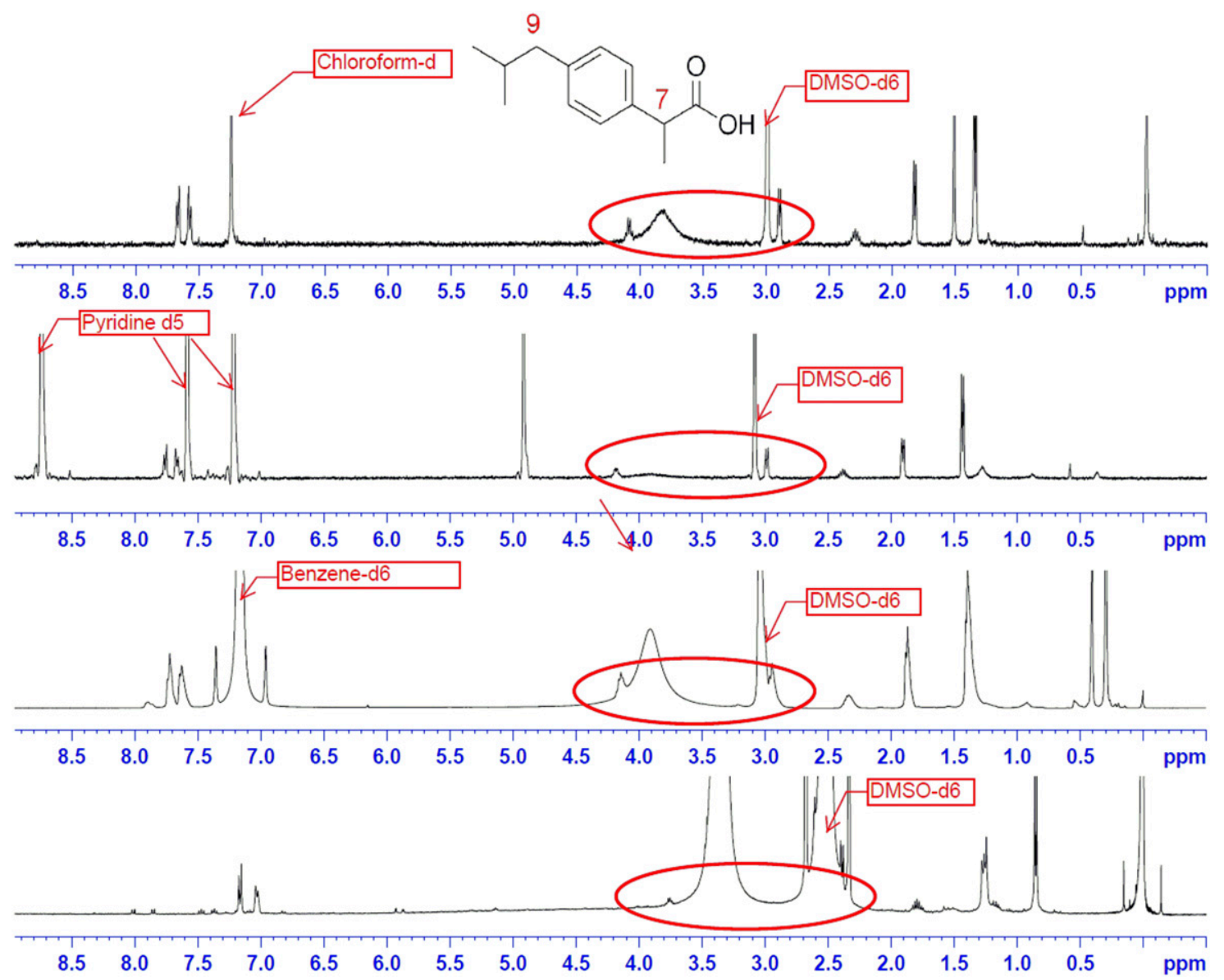

Fig. 11. ${ }^{1} \mathrm{H}$ NMR spectra of ibuprofen in different NMR solvents depicting solvent effect to minimize the interference of aliphatic region impurities.

approach $(4.59 \mathrm{mM})$ was comparable with quantification by LC-MS (4.61 mM) technique, which supports the accuracy of this method.

It was observed that due to a larger volume of solvent (DMSO-d6) in the outer tube $(\sim 600 \mu \mathrm{l})$ compared with the quantity of the sample in the capillary tube, DMSO-d6 and residual water peaks were suppressing the peak of interest from analyte in the aliphatic region. This could be a limitation of this method if a proton in aliphatic region is needed for quantification of desired metabolite. To address this concern, other deuterated solvents, such as benzene, pyridine, or chloroform, which lack aliphatic protons, could be used for sample preparation instead of DMSO, but these solvents may not be ideal if the recovered sample has to be further used as reference standard. With the hybrid capillary tube setup, however, these deuterated solvents could be used in the outer 5-mm tube without contaminating the actual sample in capillary tube. This could help to isolate the aliphatic peaks without contaminating the actual sample with undesired solvents in the outer tube. To confirm this, ibuprofen was selected as model analyte and the NMR sample was prepared in DMSO-d6. Different solvents such as benzene-d6, pyridine$\mathrm{d} 5$, and $\mathrm{CDCl}_{3}$ were used in the outer 5-mm tube, and the NMR spectra of all these samples were acquired using the same parameters (Fig. 11). Spectra acquired with benzene-d6 and pyridine-d5 in outer NMR tube showed better separation of protons 7 and 9 of ibuprofen, which were merging with water peak in DMSO-d6 data. Spectra acquired in deuterated chloroform showed clearly separated signals for $\mathrm{H} 7$ and H9, which could be used for the quantitation, if needed. This clearly indicated the effect of NMR solvent on the separation of signals from solvent peaks and suggested that this strategy could be successfully applied when there is a need for the separation of signals in the aliphatic region without contaminating the actual sample with these uncommon solvents. This could also be used if data are needed for the structural confirmation of the small quantity of crucial isolated metabolite.

In conclusion, we have developed a novel hybrid capillary tube apparatus that can be used to quantitate metabolites in the microgram level concentration with a $5-\mathrm{mm}$ room temperature NMR probe on a $400 \mathrm{MHz}$ instrument. With the use of an aSICCO signal as the internal standard, the recovered NMR sample could be further used as reference standard for biologic quantitation. At the same time, screening of different NMR solvents can be done in the outer tube to avoid contaminating the actual sample in the capillary tube or compromising the data quality. This novel setup gives the opportunity to perform metabolite characterization and quantifications with regular room temperature probes in $400 \mathrm{MHz}$ frequency spectrometers without adding any extra resources for increasing the sensitivity of the instruments.

\section{Acknowledgments}

We thank DAS (Discovery Analytical Sciences) purification team members, Venkatesh T K, Vinay Kempegowda, and Sudam Mane, for their support in collecting mass spectrometry data for the isolated material. We also thank Michael Reily (Bristol-Myers Squibb, Lawrenceville, NJ) for review of this manuscript and sharing of suggestions. We also thank Syngene International for proving all infrastructure support to do this work.

\section{Authorship Contributions}

Participated in research design: Tiwari, Ahire, Kumar, Sinha, Subramanian, Iyer, Sarabu, Bajpai.

Conducted experiments: Tiwari, Ahire, Kumar. 
Performed data analysis: Tiwari, Ahire, Kumar, Subramanian, Iyer, Sarabu, Bajpai.

Wrote or contributed to the writing of the manuscript: Chauthe, Tiwari, Ahire, Kumar, Subramanian, Iyer, Sarabu, Bajpai.

\section{References}

Annesley TM, Kurzyniec S, Nordblom GD, Buchanan N, Pool W, Reily M, Talaat R, and Roberts WL (2001) Glucuronidation of prodrug reactive site: isolation and characterization of oxymethylglucuronide metabolite of fosphenytoin. Clin Chem 47:910-918.

Barantin L, Le Pape A, and Akoka S (1997) A new method for absolute quantitation of MRS metabolites. Magn Reson Med 38:179-182.

Barding GA, Jr, Salditos R, and Larive CK (2012) Quantitative NMR for bioanalysis and metabolomics. Anal Bioanal Chem 404:1165-1179.

Bharti SK and Roy R (2012) Quantitative 1H NMR spectroscopy. Trends Anal Chem 35:5-26. Briggs RJ, Nicholson R, Vazvaei F, Busch J, Mabuchi M, Mahesh KS, Brudny-Kloeppel M, Weng N, Galvinas PA, Duchene P, et al. (2014) Method transfer, partial validation, and cross validation: recommendations for best practices and harmonization from the global bioanalysis consortium harmonization team. AAPS $J$ 16:1143-1148.

Caceres-Cortes J and Reily MD (2010) NMR spectroscopy as a tool to close the gap on metabolite characterization under MIST. Bioanalysis 2:1263-1276.

Dorado P, Berecz R, Cáceres MC, and LLerena A (2003) Analysis of diclofenac and its metabolites by high-performance liquid chromatography: relevance of CYP2C9 genotypes in diclofenac urinary metabolic ratios. J Chromatogr B Analyt Technol Biomed Life Sci 789:437-442.

Edwin DB (2000) High Resolution NMR: Theory and Chemical Applications, 3rd ed, Academic Press, New York.

Esteve V, Celda B, and Martínez-Bisbal MC (2012) Use of 1H and 31P HRMAS to evaluate the relationship between quantitative alterations in metabolite concentrations and tissue features in human brain tumour biopsies. Anal Bioanal Chem 403:2611-2625.

Farrant RD, Hollerton JC, Lynn SM, Provera S, Sidebottom PJ, and Upton RJ (2010) NMR quantification using an artificial signal. Magn Reson Chem 48:753-762.

Fischer JH, Patel TV, and Fischer PA (2003) Fosphenytoin: clinical pharmacokinetics and comparative advantages in the acute treatment of seizures. Clin Pharmacokinet 42:33-58.

Forseth RR and Schroeder FC (2011) NMR-spectroscopic analysis of mixtures: from structure to function. Curr Opin Chem Biol 15:38-47.

Huang Y, Su B-N, Ye Q, Palaniswamy VA, Bolgar MS, and Raglione TV (2014) Improving the efficiency of quantitative (1)H NMR: an innovative external standard-internal reference approach. J Pharm Biomed Anal 88:1-6.

Huttanus HM, Sheng J, and Feng X (2016) Metabolic engineering for production of small molecule drugs: challenges and solutions. Fermentation 2:4.

Johnson KA, Liu X, Huang S, Roongta V, Humphreys WG, and Shu Y-Z (2010) Rapid structure determination of microgram-level drug metabolites using HPLC-MS, fraction collection and NMR spectroscopy. Anal Methods 2:1542-1549.

Jung Y-S, Hyeon J-S, and Hwang G-S (2016) Software-assisted serum metabolite quantification using NMR. Anal Chim Acta 934:194-202

Krunić A and Orjala J (2015) Application of high-field NMR spectroscopy for characterization and quantitation of submilligram quantities of isolated natural products. Magn Reson Chem $\mathbf{5 3}$ 1043-1050

Liang Y, Ge-Ying R, Hui-Ru T, and Chao-Yang LIU (2010) Human urinary metabolite quantification in nuclear magnetic resonance-based metabonomics using electronic reference. Chin $J$ Anal Chem 38:789-794.

Molinski TF (2010) NMR of natural products at the 'nanomole-scale.' Nat Prod Rep 27:321-329.

Nagana Gowda GA and Raftery D (2015) Can NMR solve some significant challenges in metabolomics? J Magn Reson 260:144-160.

Pauli GF, Chen S-N, Simmler C, Lankin DC, Gödecke T, Jaki BU, Friesen JB, McAlpine JB, and Napolitano JG (2014) Importance of purity evaluation and the potential of quantitative ${ }^{1} \mathrm{H}$ NMR as a purity assay. J Med Chem 57:9220-9231.
Pic ÃY and Barcel ÃD (2008) The expanding role of LC-MS in analyzing metabolites and degradation products of food contaminants. Trends Anal Chem 27:821-835.

Rundlöf T, McEwen I, Johansson M, and Arvidsson T (2014) Use and qualification of primary and secondary standards employed in quantitative ${ }^{1} \mathrm{H}$ NMR spectroscopy of pharmaceuticals J Pharm Biomed Anal 93:111-117.

Saito T, Ihara T, Koike M, Kinugasa S, Fujimine Y, Nose K, and Hirai T (2009) A new traceability scheme for the development of international system-traceable persistent organic pollutant reference materials by quantitative nuclear magnetic resonance. Accred Qual Assur 14:79-86.

Scalbert A, Brennan L, Fiehn O, Hankemeier T, Kristal BS, van Ommen B, Pujos-Guillot E, Verheij E, Wishart D, and Wopereis S (2009) Mass-spectrometry-based metabolomics: limitations and recommendations for future progress with particular focus on nutrition research. Metabolomics 5:435-458.

Schadel M, Wu D, Otton SV, Kalow W, and Sellers EM (1995) Pharmacokinetics of dextromethorphan and metabolites in humans: influence of the CYP2D6 phenotype and quinidine inhibition. J Clin Psychopharmacol 15:263-269.

Schlotterbeck G, Ross A, Hochstrasser R, Senn H, Kühn T, Marek D, and Schett O (2002) Highresolution capillary tube NMR: a miniaturized 5-microL high-sensitivity TXI probe for masslimited samples, off-line LC NMR, and HT NMR. Anal Chem 74:4464-4471.

Siddiqui MR, AlOthman ZA, and Rahman N (2013) Analytical techniques in pharmaceutical analysis: a review. Arab J Chem 10:S1409-S1421.

Simmler C, Napolitano JG, McAlpine JB, Chen S-N, and Pauli GF (2014) Universal quantitative NMR analysis of complex natural samples. Curr Opin Biotechnol 25:51-59.

Spraul M, Freund AS, Nast RE, Withers RS, Maas WE, and Corcoran O (2003) Advancing NMR sensitivity for LC-NMR-MS using a cryoflow probe: application to the analysis of acetaminophen metabolites in urine. Anal Chem 75:1536-1541

Subramanian M, Paruchury S, Singh Gautam S, Pratap Singh S, Arla R, Pahwa S, Jana S, Katnapally P, Yoganand V, Lakshmaiah B, et al. (2013) Characterization of recombinantly expressed rat and monkey intestinal alkaline phosphatases: in vitro studies and in vivo correlations. Drug Metab Dispos 41:1425-1432.

Tao JA, Lin GQ, and Liese A (2009) Biocatalysis for the Pharmaceutical Industry: Discovery, Development, and Manufacturing, John Wiley \& Sons (Asia), Singapore.

Timmerman P, Blech S, White S, Green M, Delatour C, McDougall S, Mannens G, Smeraglia J, Williams S, and Young G (2016) Best practices for metabolite quantification in drug development: updated recommendation from the European Bioanalysis Forum. Bioanalysis 8:1297-1305.

Timmerman P, Lowes S, Fast DM, and Garofolo F (2010) Request for global harmonization of the guidance for Bioanalytical Method Validation and sample analysis. Bioanalysis 2:683.

Walker GS, Bauman JN, Ryder TF, Smith EB, Spracklin DK, and Obach RS (2014) Biosynthesis of drug metabolites and quantitation using NMR spectroscopy for use in pharmacologic and drug metabolism studies. Drug Metab Dispos 42:1627-1639.

Walker GS, Ryder TF, Sharma R, Smith EB, and Freund A (2011) Validation of isolated metabolites from drug metabolism studies as analytical standards by quantitative NMR. Drug Metab Dispos 39:433-440.

Weber M, Hellriegel C, Rueck A, Wuethrich J, and Jenks P (2014) Using high-performance ${ }^{1} \mathrm{H}$ NMR (HP-qNMR $\circledast$ ) for the certification of organic reference materials under accreditation guidelines-describing the overall process with focus on homogeneity and stability assessment J Pharm Biomed Anal 93:102-110.

Wieruszeski JM, Fritzinger B, Hanoulle X, Martins JC, and Lippens G (2008) Sandwich-ELISE NMR: reducing the sample volume of NMR samples. J Magn Reson 193:37-40.

Wishart DS (2008) Quantitative metabolomics using NMR. Trends Anal Chem 27:228-237.

Zhou J and Yin Y (2016) Strategies for large-scale targeted metabolomics quantification by liquid chromatography-mass spectrometry. Analyst (Lond) 141:6362-6373.

Address correspondence to: Dr. Lakshmikant Bajpai, Discovery Analytical Sciences, Biocon Bristol-Myers Squibb Research and Development Center, Syngene International, Biocon Park, Plot No. 2 \& 3, Bommasandra IV Phase, Jigani Link Road, Bangalore, India. E-mail: lakshmikant.bajpai@syngeneintl.com 